\title{
The effect of L-ornithine hydrochloride ingestion on human growth hormone secretion after strength training
}

\author{
Shinichi Demura ${ }^{1}$, Takayoshi Yamada ${ }^{2}$, Shunsuke Yamaji ${ }^{3}$, Miho Komatsu ${ }^{4}, K_{0 j i}$ Morishita $^{4}$ \\ ${ }^{1}$ Kanazawa University, Kanazawa, Japan; \\ ${ }^{2}$ Fukui National College of Technology, Fukui, Japan; \\ ${ }^{3}$ Fukui University; Fukui, Japan; \\ ${ }^{4}$ Healthcare Products Development Center, KYOWA HAKKO BIO CO., LTD. \\ Email: takay@fukui-nct.ac.jp; demura@ed.kanazawa-u.ac.jp; yamaji@u-fukui.ac.jp; miho.komatsu@kyowa-kirin.co.jp; \\ koji.morishita@kyowa-kirin.co.jp
}

Received 26 February 2010; revised 8 March 2010; accepted 10 March 2010.

\begin{abstract}
This study aimed to examine the effect of L-ornithine hydrochloride ingestion on serum growth hormone secretion response after strength training in young men who did not regularly engage in high intensity exercise. Ten healthy young males without workout habits (age: $22.2+/-1.0 \mathrm{yr}$ ). Subjects performed biceps curl strength training after L-ornithine hydrochloride and placebo ingestions. They participated in both of the above conditions randomly with a week interval in between. Serum growth hormone and ornithine levels were measured before L-ornithine hydrochloride or placebo ingestions and at $\mathbf{3 0}$ minutes after strength training. Serum growth hormone and ornithine level were measured. A change magnitude of serum growth hormone was significantly larger in the L-ornithine hydrochloride condition than in the placebo condition, and the effect size was also large $(t$ $=1.91, p=.044, E S=.75)$. A significant interaction $(F$ $\left.=\mathbf{2 8 0 . 9 8}, p=0.000, \eta_{p}{ }^{2}=0.96\right)$ was found in serum ornithine and a multiple comparison test showed that it was greater in the L-ornithine hydrochloride condition. Serum growth hormone level after strength training increases by L-ornithine hydrochloride ingestion in untrained young males.
\end{abstract}

Keywords: L-Ornithine Hydrochloride; Growth Hormone; Strength Training

\section{INTRODUCTION}

Many athletes ingest amino acids such as arginine, ornithine, methionine and lysine to make the secretion of growth hormone accelerate before training. The growth hormone is essential to gain benefits from training such as increasing muscle mass and strength due to causing anabolic action. In other words, ingestion of these special amino acids is based on the idea that training bene- fits can be effectively gained by stimulating growth hormone secretion.

Until now, the relatively larger growth hormone secretion effect (800-2200\%) by intravenous infusion of special amino acids has been found [1-3]. However, all amino acids did not always show hormone secretion effect by intravenous infusion or oral ingestion. For example, it was reported that branched-chain amino acids (BCAA) such as leucine and varine showed a slight growth hormone secretion effect (10\%), and isoleucine had no effect [1]. Moreover, it was reported also that aspartic acid, glutamic acid and cysteine stimulated little growth hormone secretion [4]. Of the amino acids which showed a growth hormone secretion effect, arginine, lysine and ornithine are believed to be superior in stimulating growth hormone secretion [3].

However, amino acids are not generally injected intravenously, but are instead ingested orally at training sites. Until now, arginine and ornithin have been mainly used to examine the effect on growth hormone secretion by oral amino acid ingestion [5-10]. However, there were mixed reports on the effect of growth hormone secretion by ornithine ingestion (negative reports: 5-9, Positive reports: 9, 10). One of the reasons for this large difference is considered to be the different ornithine amounts ingested. Subjects ingested over $170 \mathrm{mg}$ per kilogram of body mass in Bucci et al. [10] and Cynober et al. [9]'s study, who reported positive effect, but subjects ingested no more than $100 \mathrm{mg}$ per kilogram of body mass in the other studies. Moreover, subjects in all studies were mainly body builders or weight lifters who routinely conducted high intensity strength training. Muscle fibers of these subjects were larger than average people and it is inferred that a size of their muscle cells had approached the biological limit. The degree of growth hormone effect causing muscle fiber hypertrophy depends on the level of growth hormone released in the blood and the activity level of the receptors. However, in 
the body builders and weight lifters, because muscle fibers affected by growth hormone have approached the biological limit, receptor responses to an increase of growth hormone levels are small and the effect of ornithine ingestion on growth hormone secretion may be different.

Recently many people have begun conducting training for maintaining/enhancing health, and many of them actively ingest nutriceutical products to efficiently gain training benefits. Ornithine is also one of the typical nutriceutical products, and the growth hormone secretion effect has been expected by its oral ingestion. However, it has not been clarified whether a growth hormone secretion effect is also obtained in average people who do not have strenuous training habits as well as body builders and weight lifters.

This study aimed to examine the effect of L-ornithine hydrochloride ingestion on serum growth hormone secretion response after strength training in young men who do not regularly engage in high intensity exercise.

\section{METHODS}

\subsection{Subjects}

Ten healthy young adults who did not regularly engage in high intensity training participated in this study (age: 22.2 +/- $1.0 \mathrm{yr}$, height: $173.5+/-4.6 \mathrm{~cm}$, body-mass: $72.5+/-12.5 \mathrm{~kg}$ ). Written informed consent was obtained from all subjects after a full explanation of the experimental purpose and protocol. Moreover, experimental protocol in this study was approved by the inquiry committee of studies intended for humans" Kanazawa University Health \& Sports Science Ethics Committee".

\subsection{Experimental Design}

The experimental design was a double blinded cross-over method. Namely, subjects participated in both conditions of L-ornithine hydrochloride and placebo (indigestible dextrin aqueous solution) ingestions. Due to the cross-over design, all subjects participated in both conditions at the same time with washing out period of a week. The test order was also counter balanced to eliminate order effect. In addition, subjects were prohibited from intensive exercise 2 days prior to, and to fast for at least two hours before, starting exercise in order to avoid a nutritional imbalance with eating and drinking. Subjects were also instructed not to consume food or beverages that contain caffeine throughout the experimental period.

\subsection{Neutriceutical Products}

Subjects ingested L-ornithine hydrochloride or indigestible dextrin aqueous solution with the same flavor (placebo) at the ratio of $0.1 \mathrm{~g}$ per body-mass. All neutraceutical products were made by KYOWAHAKKO
BIO Co., LTD.

\subsection{Procedure}

\subsubsection{Determination of 1 Repetition Maximum}

One repetition maximum (RM) was measured for the subjects in advance in accordance with the procedure stated in Table $1[11,12]$ to determine the strength training intensity in this study.

\subsubsection{Experimental Protocol and Procedure}

Subjects entered the laboratory and consumed L-ornithine hydrochloride or a placebo after baseline blood drawing. They conducted bicep curl strength training with $60 \%$ of 1 RM intensity (Table 2 ) an hour after ingesting nutraceutical products. In addition, all sets required 10 repetitions and a 90 second rest was set between trials. The range of elbow joint motions for the concentric part was fully extended from 145 to $155^{\circ}$, flexed and means velocities for one repetition including concentric and eccentric motions were in a range of $100-150 \%$ s [13]. The second blood drawing was conducted 30 minutes after the strength training.

\subsection{Parameters}

Serum ornithine and growth hormone levels before ingestion and after strength training were measured. Changes after the strength training were subtracted from the baseline values and used for an analysis of the growth hormone.

Table 1. Procedure of 1RM measurement ${ }^{11,12}$.

(1) Warm-up with a light resistance that easily allows 5-10 repetition; provide a 1 min rest.

(2) Load increased $10-20 \%$ more than point 1 to allow 3-5 repetitions to be completed; provide a 2 min rest period.

(3) Load increased $10-20 \%$ more than point 2 to allow 2-3 repetitions to be completed; provide a $2-4 \mathrm{~min}$ rest period.

(4) Male a load increased of $10-20 \%$ more than point 3. Instruct the subject to attempt a 1RM.

(5) If the subject is successful provide a 2-4 min rest and increase the load by $5-10 \%$ increase. If the subject is unsuccessful, provide a $2-4$ min rest and decrease the load by $5-10 \%$.

(6) Continue point 5 until the subject can complete one repetition with proper exercise technique at the highest possible load.

Table 2. Strength training protocol ${ }^{13}$.

(1) Two sets of seated biceps curl at $60 \%$ of $2 \mathrm{RM}$

(2) Two sets of seated biceps curl at $60 \%$ of $1 \mathrm{RM}-1 \mathrm{~kg}$

(3) Two sets of stamding biceps curl at $60 \%$ of $1 \mathrm{RM}-2 \mathrm{~kg}$

(4) Two sets of stamding biceps curl at $60 \%$ of $1 \mathrm{RM}-3 \mathrm{~kg}$ 


\subsection{Statistical Analysis}

The difference of serum ornithine level before ingestion and at 30 minutes after strength training between both conditions was tested by a paired two way analysis of variance. A Difference of variation in serum growth hormone in both conditions was tested by a paired t-test. Effect size was calculated by partial $\eta 2$ ( $\eta \mathrm{p} 2)$ and effect size (ES). In addition, an alpha level of .05 was used for all tests.

\section{RESULTS}

Figure 1 shows the results of the paired t-test between means of serum growth hormone in both conditions. A significant difference was found between both conditions, and the L-ornithine hydrochloride condition was greater than the placebo condition and this difference was larger $(\mathrm{t}=1.91, \mathrm{p}=.044, \mathrm{ES}=.75)$. Figure 2 shows the results of the two way analysis of variance for serum ornithine level. A significant interaction $(\mathrm{F}=280.98, \mathrm{p}=$ $0.000, \eta_{\mathrm{p}}{ }^{2}=0.96$ ) was found in serum ornithine and a multiple comparison test showed that it was greater in the L-ornithine hydrochloride condition than in the placebo condition at 30 minutes after strength training. Moreover, serum ornithine level at 30 minutes after strength training was significantly greater than that before ingestion in L-ornithine hydrochloride ingestion.

\section{DISCUSSION}

The serum ornithine level at 30 minutes after strength training was greater in subjects ingesting L-ornithine hydrochloride than for subjects ingesting the placebo and this difference was large. Until now, it was reported that special amino acids such as ornthine and citruline were released and found at the peak level in the blood within 5-8 hours, being a relatively early time [8]. According to Cynober et al. [8], in the case of ornithine, blood concentration reaches a peak value at about 60 minutes after oral ingestion. Serum ornithine level after L-ornithine hydrochloride ingestion in this study was very high although 120 minutes elapsed after ingestion (about $300 \mathrm{nmol} / 1,500 \%$ increase as compared with be-fore ingestion). Increasing the amount of growth hormone level before ingestion to 30 minutes after strength training was also significantly greater in subjects ingesting L-ornithine hydrochloride than in those ingesting the placebo and this difference was large (about 200\%). Bucci et al. [10] compared blood growth hormone level before L-ornithine hydrochloride ingestion, 45 minutes and 90 minutes after ingestion under the conditions of 40 , 100 and $170 \mathrm{mg} / \mathrm{kg}$ body-mass ingestion using body builders. They reported that blood growth hormone level at 90 minutes after ingestion in the $170 \mathrm{mg} / \mathrm{kg}$ condition was significantly greater than that of the other conditions (about $150-300 \%$ ), and also significantly greater than that before ingestion (about 350\%). Although the physical characteristics and training habits of subjects, and ingestion amount of ornithine hydrochloride differed between the present study and Bucci et al. [10]'s study, growth hormone secretion effect by ornithine hydrochloride ingestion was found in both studies and the degree of secretion was also similar. Meanwhile, Lambert et al. [5] measured the blood growth hormone level every 30 minutes for 3 hours for eight male body builders with a placebo, $2.4 \mathrm{~g}$ of arginine and lysine, $1.8 \mathrm{~g}$ of ornithine and tyrosine, and $20 \mathrm{~g}$ of beef extract ingestion after 8 hours of fasting to examine the influence of amino acid such as ornithine ingestion on blood growth hormone level. They reported that little change in growth hormone level was found in any case of amino acid ingestion. Fry et al. [7] conducted a weight lifting training experiment over a week with amino acid ingestion such as ornithine hydrochloride, arginine and branched-chain amino acid together for 28 weight lifters to examine the effect of high intensity training and ingestion of amino acid neutraceutical production on endocrine response and lifting performance. As a result, an increase of growth hormone was found by training with high intensity, but not by amino acid. Although experimental protocol differed between the former study 10 and the latter two studies [5,7], considering the similarity of subjects' characteristics, a difference in their results may be caused by the kind of ingested amino acid and/or the amount ingested. Until now, growth hormone secretion effect by intravenous infusion and oral ingestion was found by amino acid such as arginine, ornithine, methionin and lysine in previous studies [1-3], but little by branched-chain amino acids such as aspartic acid, glutamic acid, cysteine, cysteine, leucine, valine and isoleucine $[1,4]$. Fogelholm et al. [6] compared blood growth hormone level over 24 hours after $2 \mathrm{~g}$ of arginine, ornithine or lysine for weight lifters to examine the effects of a small amount of amino acid ingestion on growth hormone secretion. They reported an insignificant increase of blood growth hormone level was found after ingestion of any amino acid. Considering the above, studies by Fry et al. [7] and Lambert et al. [5], used not only amino acids such as ornithine, arginine, methionine and lysine, which indicate a growth hormone secretion effect, but also the branched-chain amino acids, glutamic acid and aspartic acid, which do not indicate such a growth hormone secretion effect. The former amino acids were reduced with the whole ingestion amount being less (less than $3 \mathrm{~g}$ ). The amount administered may relate to the difference between Bucci et al. [10]'s results (over $10 \mathrm{~g}$ ) and the results of Fry et al. [7] and Lambert et al. [5].

Meanwhile, although marked growth hormone secretion was not found after $100 \mathrm{mg} / \mathrm{kg}$ body-mass ornithine hydrochloride ingestion in Bucci et al. [10]'s report, it should be noted that a significant increase was found 


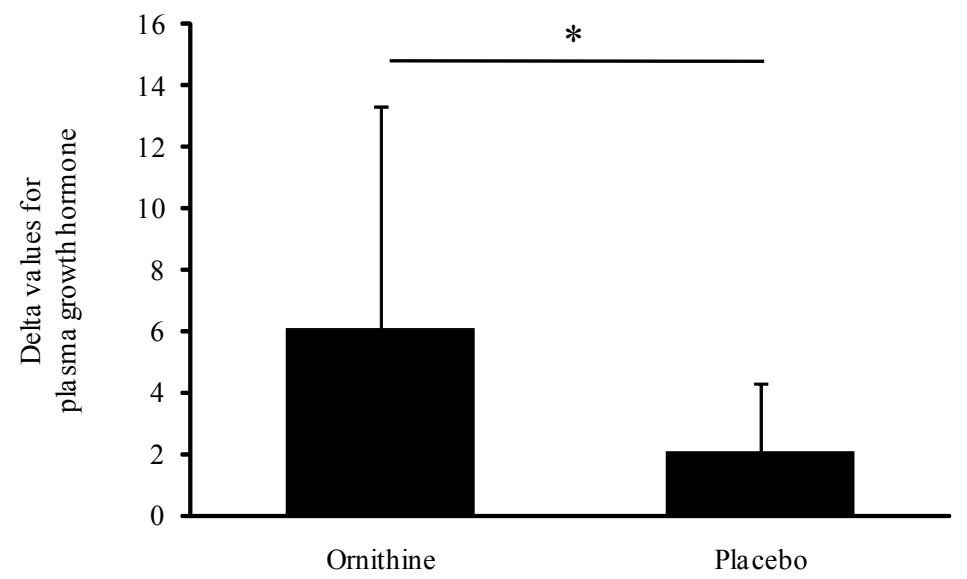

Figure 1. Variation of serum growth hormone level in both conditions*, significant difference was found between both conditions.

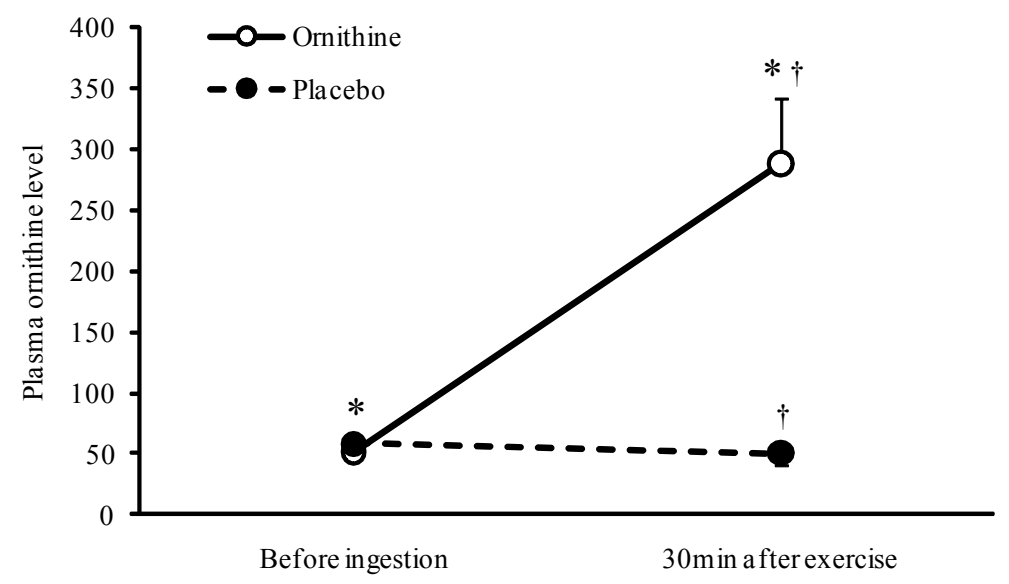

Figure 2. Serum ornithine level before ingestion and $30 \mathrm{~min}$ after strength training*, significant difference was found between before ingestion and 30 min after strengthe training, $\boldsymbol{\dagger}_{\text {significant difference was found between }}$ both conditions.

after ingestion of similar ornithine hydrochloride density in the present results. The difference between these results is inferred to be caused by the difference in the subjects' characteristics. Namely, although the present subjects had no exercise habits, the subjects in the previous studies that include Bucci et al. [10] were body builders or weight lifters who conducted strength training with high frequency and intensity. The difference in the subjects' characteristics in both studies may be related to the difference in adjustment and sensitivity of their muscle cells to training stimulation. Generally, hypertrophy of muscle fibers brought about by strength training is results from the following: effect of anabolic hormone such as growth hormone that secretes after strength training, and effect of satellite cells as stem cell existing between sarcolemmas assimirate with the existing muscle fiber $[14,15]$. Because muscle fibers of trained subjects are enlarged by repeated training stimulation, the size of muscle cells is inferred to be close to the biological limit. Strength of growth hormone effect regarding muscle fibers hypertrophy depends on the growth hormone level released into the blood and activity level of the receptors. However, responses of receptors to an increase of growth hormone level are inferred to be small in muscle fibers nearly enlarged to the biological limit. Namely, muscle hypertrophy of the trained subjects depends on the differentiation and disintegration of satellite cells with training stimulation rather than anabolic hormone secreted after training. Therefore, the present results may have been different from Bucci et al.'s results in spite of the same ornithine hydrochloride ingestion. Meanwhile, in the case of the present subjects without strength training habits, because activity level of receptors increases with an increase of blood growth 
hormone level, adjustment to strength training may occur relatively smoothly. In addition, because the hypertrophy regarding satellite cells is expected, training effects can be obtained efficiently. From the above, it is considered that even in subjects without an exercise habit, a growth hormone secretion effect was obtained by ingestion of ornithine hydrochloride in lower amounts than the previous study (170 $\mathrm{mg} / \mathrm{kg}$ body-mass) [10], which was reported to obtain a growth hormone secretion effect. However, Collier et al. [16] reported that gastrointestinal discomfort is often caused by special amino acids such as ornithine and arginine and the degree of this discomfort is related to the amount ingested. Although a growth hormone secretion effect was found by a lower ingestion amount as compared with Bucci et al. [10]'s report in this study, six out of ten subjects complained of gastrointestinal discomfort such as stomachache and diarrhea. Further studies may be required to examine the lower amount of ingestion that obtains growth hormone secretion effect without such gastrointestinal discomfort.

\section{CONCLUSIONS}

Blood growth hormone level increases after strength training by L-ornithine hydrochloride ingestion in untrained males.

\section{ACKNOWLEDGEMENT}

This work was supported by a KYOWA HAKKO BIO CO., LTD.

\section{REFERENCES}

[1] Knopf, R.F., Conn, J.W., Fajans, S.S., Floyd, J.C., Guntsche, E.M. and Rull, J.A. (1965) Plasma groeth hormone response to intravenous administration of amino acids. The Journal of Clinical Endocrinology and Metabolism, 25, 1140-1144.

[2] Evain-Brion, D., Donnadieu, M., Roger, M. and Job, J.C. (1982) Simultaneous study of somatotrophic and corticotrophic pituitary secretions during ornithine infusion test. Clinical Endocrinology, 1982, 17, 119-122.

[3] Chromiak, J.A. and Antonio, J. (2002) Use of amino acids as growth hormone-releasing agents by athletes. Nutrition, 18, 657-661.

[4] Carlson, H.E., Miglietta, J.T., Roginsky, M.S. and Stegink, L.D. (1989) Stimulation of pituitary hormone secretion by neurotransmitter amino acids in humans. $\mathrm{Me}$ tabolism, 38, 1179-1182.
[5] Lambert, M.I., Hefer, J.A., Millar, R.P. and Macfarlane, P.W. (1993) Failure of commercial oral amino acid supplements to increase serum growth hormone concentrations in male body-builders. International Journal of Sport Nutrition, 3, 298-305.

[6] Fogelholm, G.M., Näveri, H.K., Kiilavuori, K.T. and Härkönen, M.H. (1993) Low-dose amino acid supplementation: no effects on serum human growth hormone and insulin in male weightlifters. International Journal of Sport Nutrition, 3, 290-297.

[7] Fry, A.C., Kraemer, W.J., Stone, M.H., Warren, B.J., Kearney, J.T., Maresh, C.M., Weseman, C.A. and Fleck SJ. (1993) Endocrine and performance responses to high volume training and amino acid supplementation in elite junior weightlifters. International Journal of Sport Nutrition, 3, 306-322.

[8] Cynober, L. (2007) Pharmacokinetics of arginine and related amino acids. Journal of Nutrition, 137, 1646-1649.

[9] Cynober, L., Coudray-Lucas, C., de Bandt, J.P., Guéchot, J., Aussel, C., Salvucci, M. and Giboudeau, J. (1990) Action of ornithine alpha-ketoglutarate, ornithine hydrochloride, and calcium alpha-ketoglutarate on plasma amino acid and hormonal patterns in healthy subjects. Journal of the American College of Nutrition, 9, 2-12.

[10] Bucci, L., Hickson, J.F., Pivarnik, J.M., Wolinsky, I., Mc Mahon, J.C. and Turner, S.D. (1990) Ornithine ingestion and growth hormone release in bodybuilders. Nutrition Research, 10, 239-245.

[11] Ball, N. and Scurr, J. (2010) An assessment of the reliability and standardisation of tests used to elicit reference muscular actions for electromyographical normalisation. Journal of Electromyography and Kinesiology, 20, 81-88.

[12] Earle, R.W. (1999) Weight training exercise prescription. Essentials of Personal Training Symposium Workbook. SCA Certification Commission, Lincoln.

[13] Hansen, S., Kvorning, T., Kjaer, M. and Sjøgaard, G. (2001) The effect of short-term strength training on human skeletal muscle: the importance of physiologically elevated hormone levels. Scandinavian Journal of Medicine \& Science in Sports, 11, 347-354.

[14] Chesley, A., MacDougall, J.D., Tarnopolsky, M.A., Atkinson, S.A. and Smith, K. (1992) Changes in human muscle protein synthesis after resistance exercise. Journal of Applied Physiology, 73, 1383-1388.

[15] White, T.P. and Esser, K.A. (1989) Satellite cell and growth factor involvement in skeletal muscle growth. Medicine and Science in Sports and Exercise, 21, 158-163.

[16] Collier, S.R., Casey, D.P. and Kanaley, J.A. (2005) Growth hormone responses to varying doses of oral arginine. Growth Hormone \& IGF Research, 15, 136-139. 